\title{
System Architecture of the BCU Payload on Tatiana-2
}

\author{
Shyh-Biau Jiang ${ }^{1,2, *}$, Tse-Liang Yeh ${ }^{1,2}$, Huey-Ching Yeh ${ }^{3}$, Jann-Yeng Liu ${ }^{3}$, Ying-Hao Hsu ${ }^{2}$, \\ and Li-Yeh Liu ${ }^{1}$ \\ ${ }^{1}$ Institute of Mechanical Engineering, National Central University, Jhongli, Taiwan, ROC \\ ${ }^{2}$ Institute of Opto-Mechatronics Engineering, National Central University, Jhongli, Taiwan, ROC \\ ${ }^{3}$ Institute of Space Science, National Central University, Jhongli, Taiwan, ROC
}

Received 8 August 2011, accepted 20 September 2011

\begin{abstract}
In conjunction with the international collaborative project of ESEMS (Experimental Scientific Education Micro Satellite) whose goal is to develop an experimental scientific-education microsatellite with science payloads, a team consisting of professors and students from the National Central University (NCU) has designed and fabricated a set of space flight instruments for space weather studies. The Block of Central University (BCU) made payload has been flown successfully on board Tatiana-2. To our knowledge, the BCU payload is the very first successful satellite payload which has been developed from design and component selection to the completion of the flight module mainly by students and faculty on the NCU campus in Taiwan. This paper describes some details of the engineering effort in building the BCU payload, including sensing devices (ETP and MRM), data processing unit, and power supply. Samples of flight data acquired by BCU are also presented to show that all units of the BCU system and payload-spacecraft interfaces functioned well as expected. The flight data provides direct evidence that the NCU team is capable of developing spaceflight quality instruments for future satellite missions.
\end{abstract}

Key words: Tatiana-2, Ionosphere, BCU payload, Electron Temperature Probe (ETP), Magneto-Resistive Magnetometer (MRM), Flight software, Taiwan

Citation: Jiang, S. B., T. L. Yeh, H. C. Yeh, J. Y. Liu, Y. H. Hsu, and L. Y. Liu, 2012: System architecture of the BCU payload on Tatiana-2. Terr. Atmos. Ocean. Sci., 23, 193-208, doi: 10.3319/TAO.2011.09.20.01(AA)

\section{INTRODUCTION}

Tatiana-2 is one of the ESEMS (Experimental Scientific Education Micro Satellite) microsatellites, and was launched on 17 September 2009 into a pre-noon $(8.93$ LT) to pre-midnight sun synchronous orbit at an altitude of $\sim 827$ - $836 \mathrm{~km}$ with an orbital inclination angle of $98.785^{\circ}$ (Kalegaev 2009). Unfortunately, the Tatiana- 2 satellite was decommissioned on 12 February 2010 due to an orientation system failure (MsuNews 2010).

Because Tatiana-2 is a sun-synchronous satellite in the topside ionosphere, the NCU team originally proposed a cluster (suite) of three sensors including an auroral electron energy spectrometer (EES), an electron temperature probe (ETP) and a high sampling magneto-resistance magnetometer (MRM) to investigate space weather features as well as earthquake induced signatures (e.g., Oyama et al. 2008)

\footnotetext{
* Corresponding author

E-mail:sbjiang@viewmove.com
}

in the terrestrial ionosphere. The proposed instruments are commonly used to measure ionospheric plasma dynamics and magnetic variations induced by field-aligned currents (Yeh et al. 2008a).

In addition to identifying the proper sensor set for science, what we were most concerned with were the various interfaces between payload and satellite such as communication, power supply and data handling, since this was our first payload designed for use aboard a Russian satellite. From an engineering point of view, the communication interface design must be simple but requires a package of testing tools for monitoring the exchange of bidirectional packets during the satellite-payload integration test. All errors of communication between satellite and payload could be found exactly and instantly through the package to improve the efficiency of test a lot. Therefore, we can reduce the difficulties due to the absence of interface specification details and experience in handling a satellite-payload integration test; moreover, we can minimize miscommunication 
between NCU and Russian engineers due to language challenges and cultural differences.

To simplify the power supply interface, the cluster has its own switching DC-DC converters. The function of these converters is to covert the most commonly used satellite voltage $28 \sim 34 \mathrm{~V}$ to a voltage of \pm 5 and $12 \mathrm{~V} \mathrm{DC}$ for internal use of the BCU sensors. The most important design feature of the NCU cluster is its own flight computer as a data processing unit (DPU) to simplify the data handling interface for the signal sensing device to read, and increase payload flexibility of the satellite. In addition to controlling the instruments' experiments and collecting flight data, the NCU flight computer also provides a serial communication port and protocol. Therefore, the Tatiana- 2 can command a payload experiment and then download the experimental data. To speed up satellite-payload integration testing and reduce travel and personnel costs, the NCU team has also developed its own satellite-payload communication interface monitoring module (Hsu et al. 2008 and Yeh et al. $2008 \mathrm{~b}$ ). The module can monitor the satellite commands as well as the payload responses, and displays them on a PC to assist both the satellite and payload engineers to clarify the causes of communication errors, thus, accelerating the debugging processes.

In this paper, section 2 describes the overall configuration of the BCU payload. Section 3 briefly introduces the principles and functions of the sensors. The design principles and the operation modes of the BCU support system are described in section 4 . Some flight data collected by BCU are presented in section 5. Final section provides concluding comments.

\section{GENERAL CONFIGURATION OF THE BCU PAYLOAD}

Figure 1 shows the front and back view of the payload. The BCU circuits and most of the sensors were packaged in the same enclosure (payload case), which is an aluminiumalloy chassis, $150 \mathrm{~mm} \times 150 \mathrm{~mm} \times 85 \mathrm{~mm}$. The ETP electrode is mounted at the end of a $32.4 \mathrm{~cm}$ probe rod extending upward from the top wall of the front panel of the chassis. The power and communication connectors to the satellite are located in the rear panel of the chassis. The coordinates of the $\mathrm{BCU}$ chassis are defined as that positive $\mathrm{Z}$ directs from the rear panel to the front panel, positive $Y$ directs upward and the direction of positive $\mathrm{X}$ is determined by the right-hand orthogonal coordinate system.

The payload case is divided into two layers. The EES and the power converter are housed in the back layer. EES has been designed to have an upward facing entrance to allow precipitating electrons access from the top. However, because of the weight constraint, the EES was removed from the BCU flight module, so the opening for a particle entrance was sealed. The front layer contains the data processing unit, the ETP signal processing circuit, the MRM circuit, and the program updating circuit. The ETP electrode is a circular disk mounted in the $\mathrm{X}$-Y plane with its normal vector pointing to the positive $\mathrm{Z}$ direction.

The circular disk is divided into two semi-circular sensors with a $10 \mathrm{~mm}$ gap, which are fixed to the probe rod as shown in Fig. 1. (The polarities of the XYZ coordinates for the MRM sensors are different from those of the BCU chassis. Both have the same positive direction for the $\mathrm{Z}$ axis,
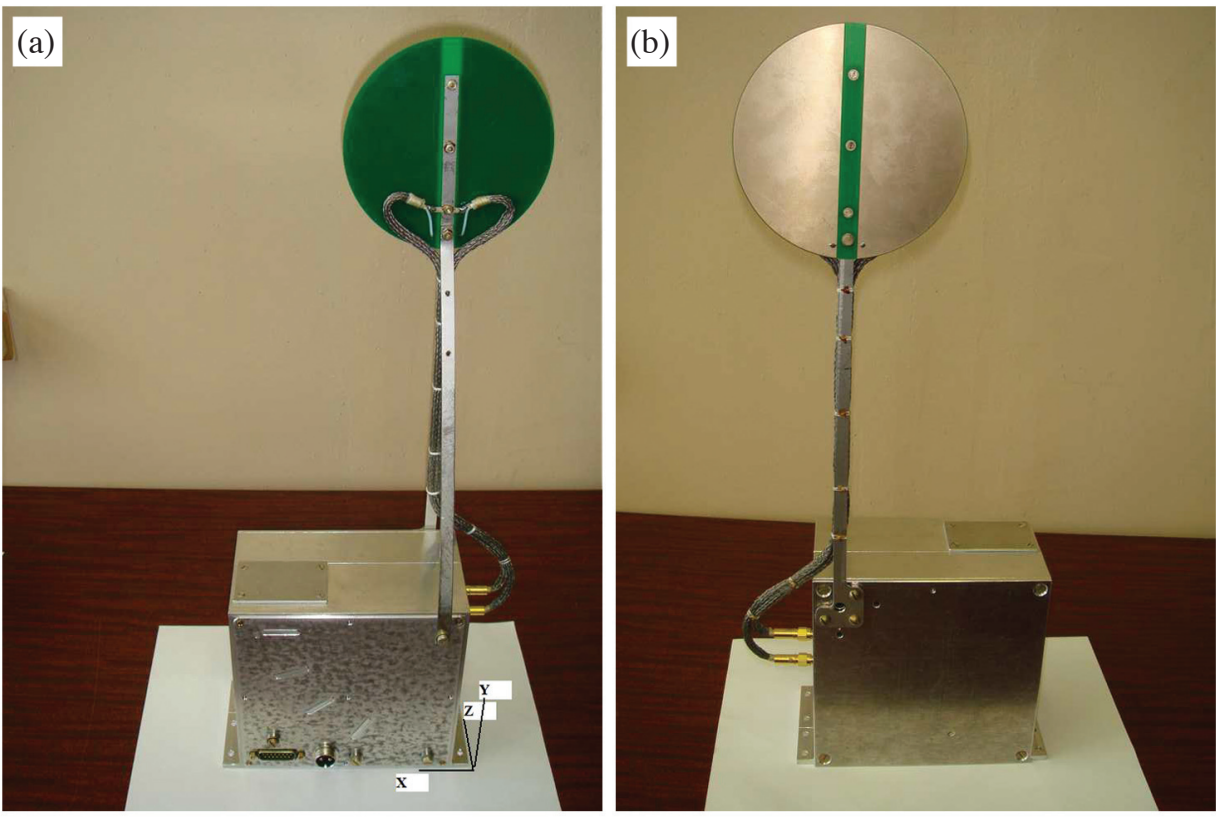

Fig. 1. Front view (b) and rear view (a) of the BCU payload. In the front view, the left hemisphere of the ETP probe is the reference electrode " $\mathrm{P}_{\text {ref" }}$ " and the right is the driven measurement electrode "P." 
but have opposite directions for the positive $\mathrm{X}$ and $\mathrm{Y}$ coordinates.) In addition to the main sensors, each side of the $\mathrm{BCU}$ case has a temperature sensor to maintain a constant temperature inside the chassis. Excluding the EES, the net weight of the BCU payload is $1.6 \mathrm{~kg}$. Except for the ETP electrode, the BCU payload is covered by the insulation jacket provided by Russia colleagues. As shown in Fig. 2, the BCU was mounted at the top of the front-end Tatiana-2 platform since the ETP is designed to view along the satellite ram direction.

As mentioned, the development of the BCU payload is our first project in collaboration with a Russian institute. To minimize travel expenses and the difficulties in mutually understanding the various interface specifications and requirements due to language challenges, we have designed our own DPU system and DC power conversion circuit to simplify the satellite interfaces. We use only a single power input and a single serial port to communicate with the satellite. At the same time, a program loader was included in the BCU payload, so our engineers can update the BCU system as needed, even when travelling in Russia. Moreover, we have developed communication tools (Liu et al. 2012) to help monitoring the communication details between the satellite and the BCU payload. As a result, we can save a lot of time and travel costs for the debugging processes during the integration tests of a payload and satellite.

The functional block diagram of the BCU payload is given in Fig. 3. The BCU is composed of DC/DC power converters, the main instruments, ETP, MRM and EES (only to the design stage), a BCU DPU computer, a program loader (PL), and a set of temperature sensors. Peripheral support system includes the Communications Monitor (CM) and a Personal Computer (PC). The electrical interfaces to Tatiana-2 are through a single power input and a single serial communication port (RS422). The BCU DC / DC converters convert the $28 \sim 34 \mathrm{~V}$ DC into $+5,-5$, and $12 \mathrm{~V}$ as required by the payload. Via the RS422 communications, the BI (block of information) computer of Tatiana-2 issues instructions and gets responses to/from the BCU computer.
The purpose of the CM is to monitor the RS422 communications between the Tatiana- 2 and the BCU-DPU computers. The data acquired by the $\mathrm{CM}$ is sent to the $\mathrm{PC}$, which forms the reference base for the staff members to handle the debugging during the satellite-payload communication test. The PC computer can also, via RS422, communicate with the program loader that reads and writes programs from/into

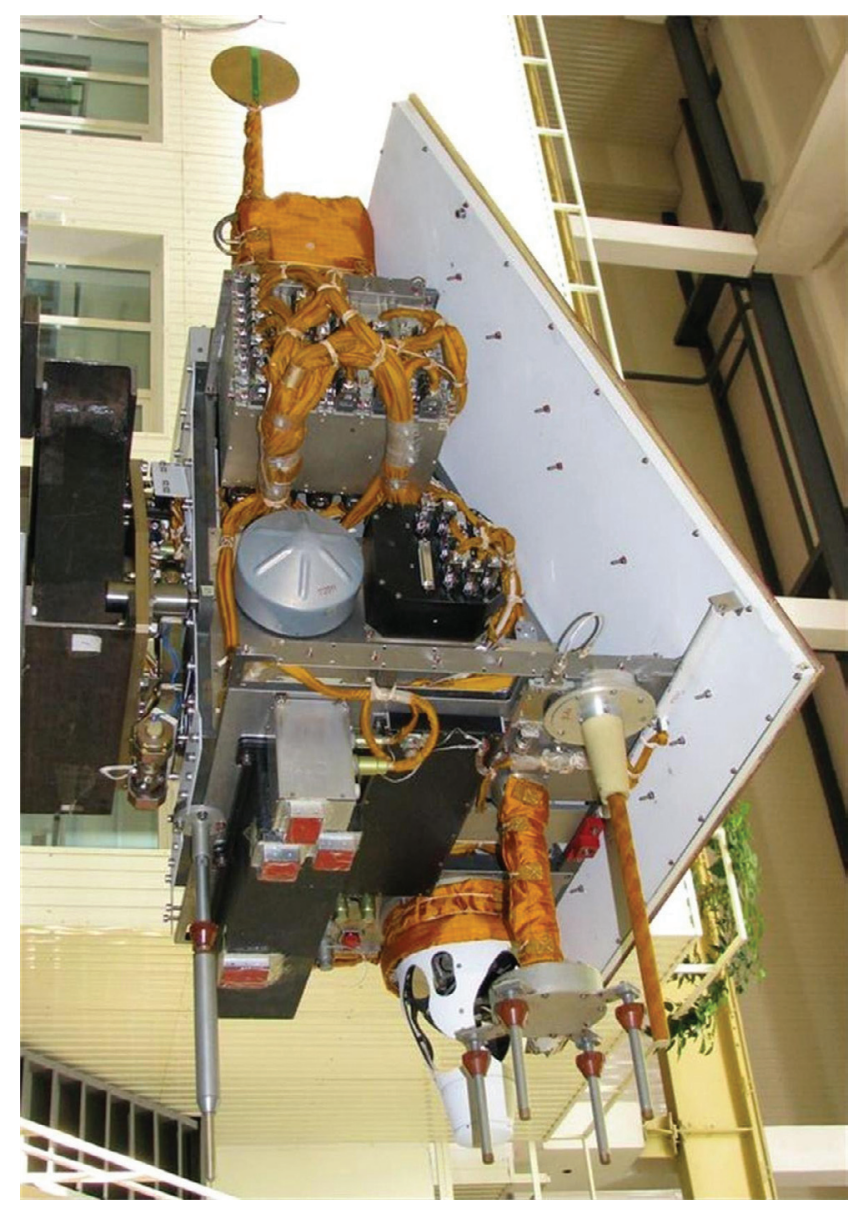

Fig. 2. A photograph of the integrated Tatiana-2 satellite and payloads (Courtesy of VNIIEM).

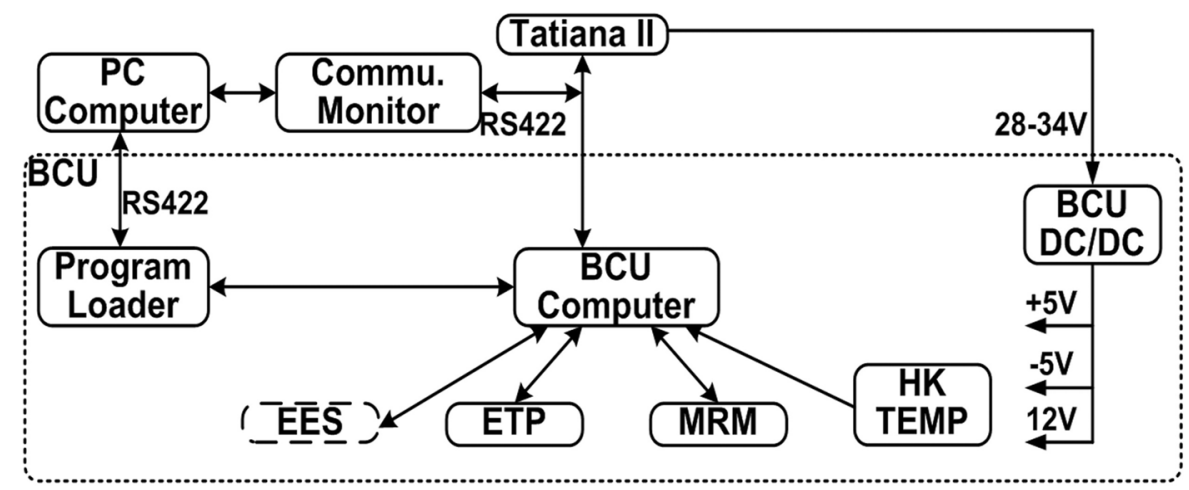

Fig. 3. The functional block diagram of the BCU and its supporting systems. 
the BCU-DPU program memory. Program Loader can help facilitate the updating of the firmware development.

As satellite telemetry is limited in certain areas over ground stations, the data acquired by the $\mathrm{BCU}$ during its experiments along the orbits will be saved to the memory buffer and are downloaded when the satellite enters into the field of view of a receiving ground station. Furthermore, since the BCU payload has a number of instruments, signals from them will inevitably interfere with each other through the circuit or radio waves. It is therefore necessary to allocate properly the experimental time for all the equipments in order to obtain a cleaner signal. Therefore, each instrument is subject to the following three steps during the measurement process: (1) entering the instrument measurement mode; (2) waiting for a settled signal; and (3) acquiring the data. The details of how the DPU computer handles the time allocation and the time line of operations for each instrument will be discussed in the later sections.

\section{DESCRIPTION OF SENSORS}

This section briefly describes the main functions and physical principles of the sensors constituting the BCU payload. Detailed characteristics of the data acquired by each of the sensors and their scientific applications will be discussed elsewhere.

\subsection{Electron Temperature Probe (ETP)}

The electron temperature probe (ETP) was invented by K. Hirao (Hirao 1970a, b) and improved by K. Oyama in Japan in early 1970 . Since then the ETP probe has become a popular instrument for measuring electron temperatures in the ionosphere. The probe has been installed on many of international satellites because the operation of ETP is less sensitive to the potential sheath structure enveloped by its electrodes (Oyama 1976).

In general, when an electrode is floating in the ionosphere, a negative potential drop will be developed at the electrode with respect to the ambient plasma. This is because the electrons have higher thermal speed than positive ions and will reach the electrode faster than ions. As a result, a positively charged plasma sheath is formed in front of the negatively charged electrode. Since the configuration of a positive plasma sheath and a negative electrode is similar to the PN junction of a diode, the current/voltage relationship between the plasma and the electrode is similar to that of a diode, which is shown in the far right curve of Fig. 4a. On the I-V curve the probe voltage where the sum of the ion and electron currents is zero is called the floating potential $\left(\mathrm{V}_{\mathrm{f}}\right)$. When a sine wave (AC) voltage (e.g., $30 \mathrm{kHz}$ ) is applied to the probe as indicated in Fig. $4 \mathrm{~b}$, more electron current is collected $(\Delta \mathrm{I}-)$ during the positive phase of the sine wave than ion current collected $(\Delta \mathrm{I}+)$ during the negative phase.
Thus the probe will accumulate negative charge until a new floating potential $\left[\mathrm{V}_{\mathrm{f}-\mathrm{ac}+\mathrm{dc}}\right]$ is reached, which is shifted negatively with respect to the original floating voltage $\left(\mathrm{V}_{\mathrm{f}-\mathrm{dc}}\right)$. It has been shown that the floating voltage shift $\left(\Delta \mathrm{V}_{\mathrm{f}}=\mathrm{V}_{\mathrm{f}-\mathrm{ac}}\right)$
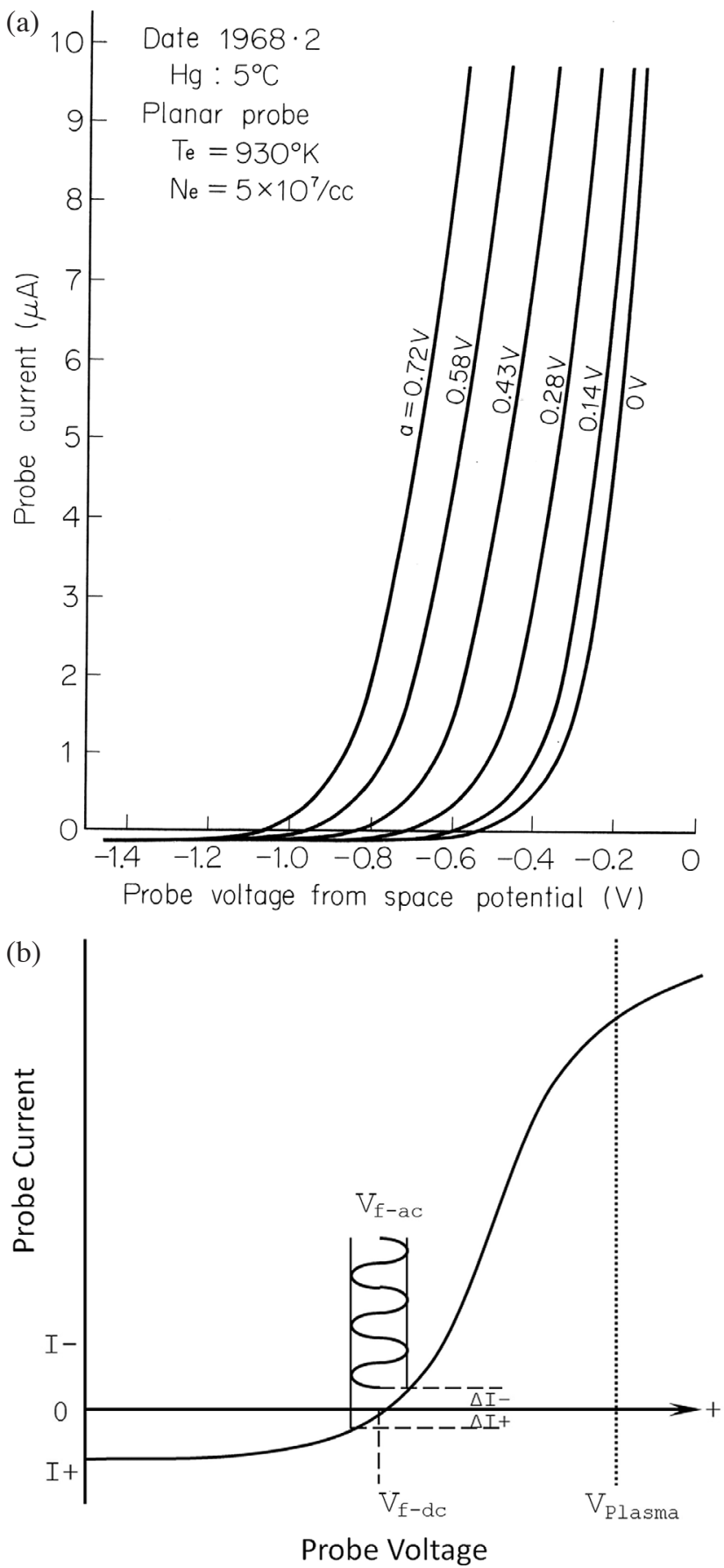

Fig. 4. (a) The current-voltage (I-V) curves of ETP in plasma. The floating potential shifts negatively with increasing amplitude [a] of a driving sinusoidal signal superposed onto the probe DC voltage. The far right curve indicates the I-V relation for the case with zero driving AC voltage (Courtesy of Oyama 2008). (b) The negative shift of floating potential $\left[\mathrm{V}_{\mathrm{f}-(\mathrm{dc}+\mathrm{ac})}\right]$ resulting from the super-position of a sine wave voltage on the DC probe voltage $\left(\mathrm{V}_{\mathrm{f}-\mathrm{dc}}\right)$ with respect to ambient plasma, due to $|\Delta \mathrm{I}-|>|\Delta \mathrm{I}+|$. 
depends on the amplitude, a, of the AC voltage and the electron temperature $\left(T_{e}\right)$. For the case of fixed $T_{e}$, Fig. 4a shows that the larger the $A C$ voltage is, the more the $V_{f}$ shifts negatively. By comparing the ETP floating voltage shift of a $=0.25 \mathrm{~V}$ AC voltage with that of $0.5 \mathrm{~V}$ AC voltage, the electron temperature can be calculated if we assume that the distribution of ambient plasma in the phase space is Maxwellian.

Figure 5 depicts the functional elements of the BCUETP circuit system. The system includes two electrodes (plates $\mathrm{P}$ and $\mathrm{P}_{\mathrm{ref}}$ ), AC generator, resistors, capacitors and three operational amplifiers (OPs). Figure 6 is a functional block diagram of the circuit. As the reference electrode passes through the plasma, a voltage difference with respective to ambient plasma $\left(\Delta \mathrm{E}_{\text {sheath }}\right)$ is generated. When this largely negative potential is applied to the referenced electrode, the voltage difference $\left(\Delta \mathrm{E}_{\text {sheath }}\right)$ drives a current, $\boldsymbol{i}_{\mathrm{dc}}$, through the plasma-electrode junction. The current charges the capacitor (or RC circuit) to increase the reference-electrode voltage until the balance of the current (zero current) is reached. Then, the voltage of the referenced electrode defines the DC floating voltage $\left(\mathrm{V}_{\mathrm{f}-\mathrm{dc}}\right)$.

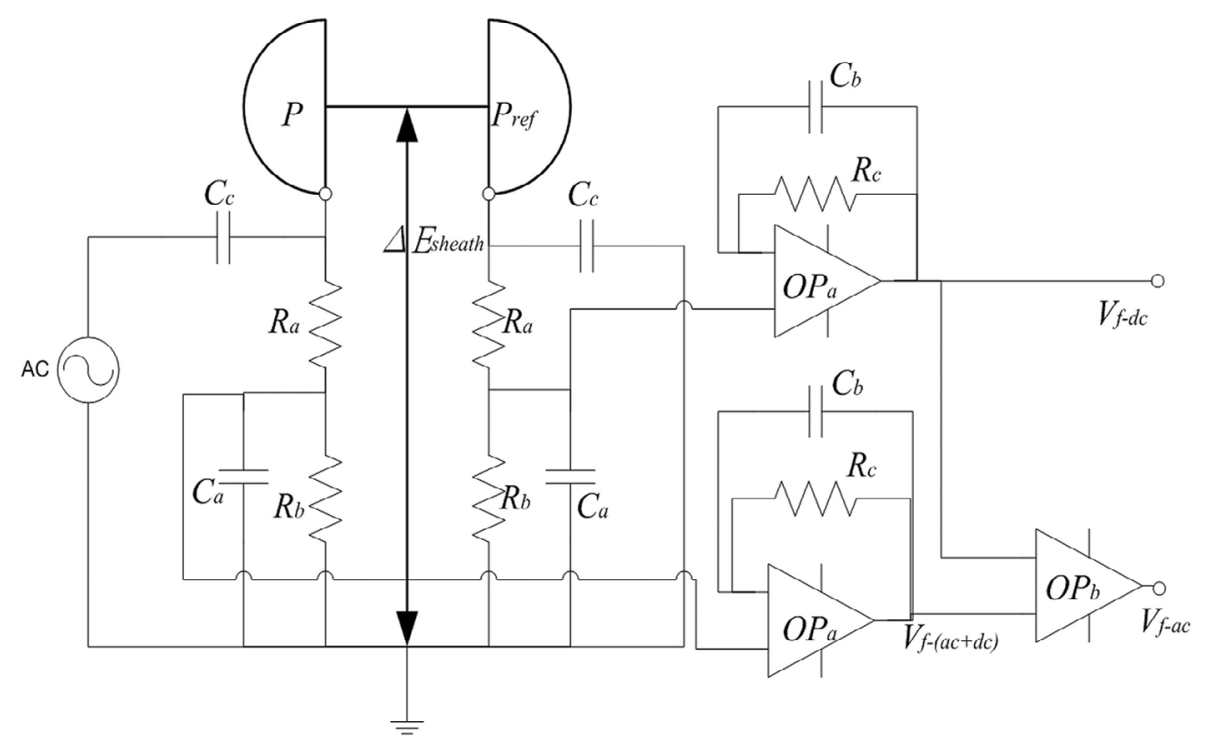

Fig. 5. The electrometer amplifying circuitry of BCU-ETP.

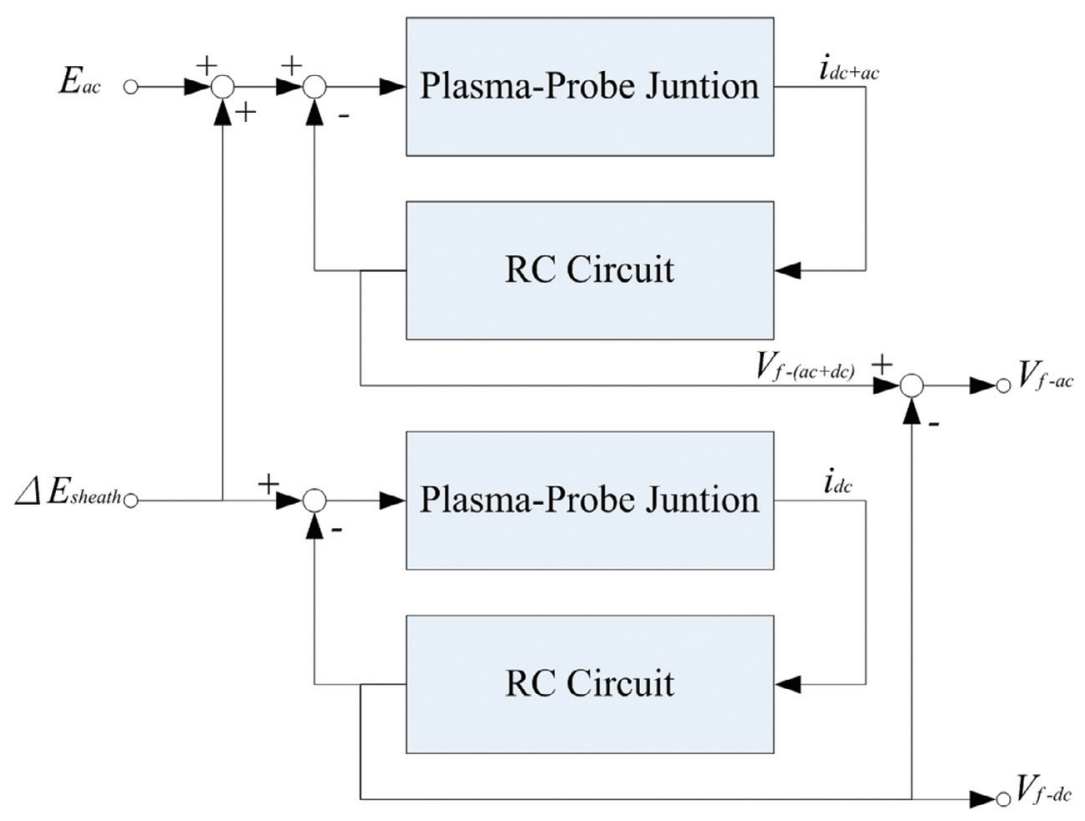

Fig. 6. Block diagram of the ETP circuit. 
In addition to $\Delta \mathrm{E}_{\text {sheath }}$, an $\mathrm{AC}$ voltage $\mathrm{E}_{\mathrm{ac}}$ is superimposed on the other electrode (i.e., the measurement-electrode plate). Again the net voltage difference drives the $i_{(\mathrm{dc}+\mathrm{ac})}$ current through the plasma-electrode junction and charges the capacitor RC circuit until reaching another floating (balance) voltage $V_{f-(d c+a c)}$. The difference between $V_{f-(d c+a c)}$ and $\mathrm{V}_{\mathrm{f} \text { - dc }}$ is the AC floating voltage shift $\mathrm{V}_{\mathrm{f}-\mathrm{ac}}$. For the BCUETP we fix the frequency at $30 \mathrm{kHz}$, but vary the $\mathrm{E}_{\mathrm{ac}}$ in three phases which are $0.5,0.25$, and $0 \mathrm{~V}$, respectively, to measure the floating voltage shifts. The time duration for each phase takes $150 \mathrm{~ms}$ since after each change of the AC amplitude it is required to wait for the signal to become stable before the measurements are taken. Post-processes enable the electron temperatures to be derived from the measurements of the floating voltage shifts. However, the AC current can induce electromagnetic radiation, which may cause electromagnetic interference to the other circuits. We therefore need to carefully arrange the timing of the ETP AC-voltage excitation as well as the data taking in order to complement with the other instruments.

Here we present the formula that relates the electron temperature to the measured floating potential shift $\left(\Delta \mathrm{V}_{\mathrm{f}}\right)$ in Eq. (1). The various values of $\Delta \mathrm{V}_{\mathrm{f}}$ can be obtained by varying the amplitude [a] of the $\mathrm{AC}$ voltage superimposed on the measurement-electrode plate (Oyama 2008).

$\Delta \mathrm{V}_{\mathrm{f}}=\left(-\mathrm{k} \mathrm{T}_{\mathrm{e}} / \mathrm{e}\right) \cdot\left\{\mathrm{l}_{\mathrm{n}}\left[\mathrm{I}_{0}\left(\mathrm{ea} / \mathrm{k} \mathrm{T}_{\mathrm{e}}\right)\right]\right\}$

where $\mathrm{I}_{0}(\mathrm{x})$ is a modified Bessel Function of zero-th order, $l_{n}$ is natural logarithmic, e is elementary charge, and $\mathrm{k}$ is Boltzmann constant.

Note, Eq. (1) is applicable under the assumption that the phase-space distribution of ambient electrons can be characterized by a single Maxwellian distribution function. In the case of our ETP measurements the electron temperature can be derived from Eq. (1) using either $\Delta \mathrm{V}_{\mathrm{fl}}$ at a $=0.25$ $\mathrm{V}$ alone, $\Delta \mathrm{V}_{\mathrm{f} 2}$ at a $=0.5 \mathrm{~V}$ alone, or the ratio of $\Delta \mathrm{V}_{\mathrm{f} 1} / \Delta \mathrm{V}_{\mathrm{f} 2}$. If the electron distribution is indeed Maxwellian, the three estimates of temperature should be consistent. If they are much different, the plasma is considered to be non-Maxwellian or dominated by high energy electrons.

\subsection{Anisotropic Magneto-Resistive Magnetometer}

Anisotropic Magneto-resistive (AMR) component is made of thin film of nickel-iron (Permalloy) deposited on a silicon wafer and is patterned as a resistive strip (Caruso et al. 1999). The resistance of an AMR resistor varies with an ambient magnetic field and temperature. However, the percentage change of a single AMR resistor is limited to about $2 \% \sim 3 \%$ in the presence of a magnetic field. To improve the sensitivity and prevent a temperature disturbance, our magnetic sensor is composed of four magnetic resistors arranged as a Wheatstone bridge (shown in Fig. 7a). Unlike a flux gate magnetometer, which is a transformer type device and therefore requires basic sinusoidal excitation, the magnetic-resistive type magnetometer can have higher response frequency that is not limited by the fundamental excitation frequency. The BCU system uses three identically designed AMR sensors to measure the three-dimensional vector components of the Earth's magnetic field. The AMR sensor chip has maximum resolution of $3 \mathrm{nT}$ and frequency response of $5 \mathrm{MHz}$ (Honeywell 2000). However, limited by communication bandwidth and resolution of ADC components in use, the finalized 3D magneto-resistive magnetometer (MRM) in $\mathrm{BCU}$ has a resolution of $23 \mathrm{nT}$, and a sampling frequency of $2.22 \mathrm{~Hz}$. The statistical property in the measurement was investigated to determine if it was possible to improve the resolution towards sub $\mathrm{nT}$ at $\mathrm{kHz}$ sampling frequency as required by a specific scientific application.

A disadvantage of the AMR magnetometer is that the performance of an anisotropic magnetic resistor degrades with increasing entropy in its residual magnetic field. Generally, it is most stable when the magnetic field orientations of the grains in the resistor strip are randomly distributed. Nevertheless, when all the grains have the same residual magnetic field direction, an AMR has the highest sensitivity. A way to overcome this deficiency is to magnetize the sensor frequently to unify the residual magnetic orientations. By this way, not only the sensitivity along its sensitive-axis direction is enhanced but also the effect of cross-axis direction is suppressed. As the AMR sensors that constitute Wheatstone bridge may not be balanced, so the output voltage contains bridge offset. Most of the bridge offset can be cancelled by applying a fixed voltage with different polarity to the offset. The rest of the bridge offset can also be eliminated through a "Set-Reset" scheme, which combines sensor magnetization and offset compensation. This scheme measures the magnetic field two times, one after positive magnetization (called "Set") and the other after negative magnetization (called "Reset"), and then subtracts the second measured value from the first to eliminate the offset.

As shown in Fig. 7b, the Set-Reset generator is synchronized to the sampling cycle to generate Set-Reset current to magnetize AMR sensors. Each Set or Reset cycle needs approximately $60 \mu \mathrm{s}$ after the pulses to get the stabilized signals. So that if it makes one measurement per Set-Reset cycle, a MRM system theoretically can reach the highest sampling rate of $8 \mathrm{kHz}$. Moreover, this $2 \mu \mathrm{s}, 4 \mathrm{amps}$, instantaneous pulse current may introduce electromagnetic interference to other devices. To minimize the impact to the measurement data of other equipment, MRM must be coordinated with other instrument for measuring time. As shown in the block diagram, a magnetic field is converted into voltage through the AMR sensor, amplified by AMP\&LPF block, compensated by the DAC, and then finally sampled as data by the ADC. Taking the impact of temperature on the AMR into consideration, we have arranged a tempera- 
(a)

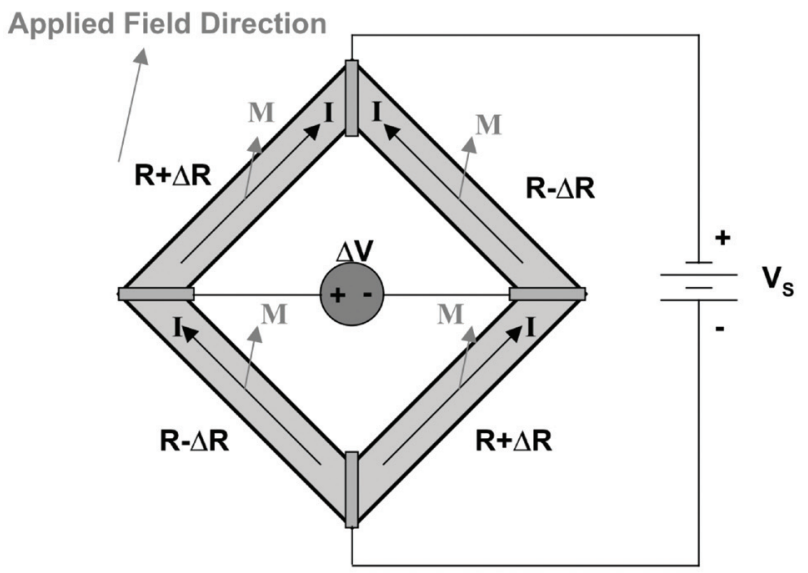

(b)

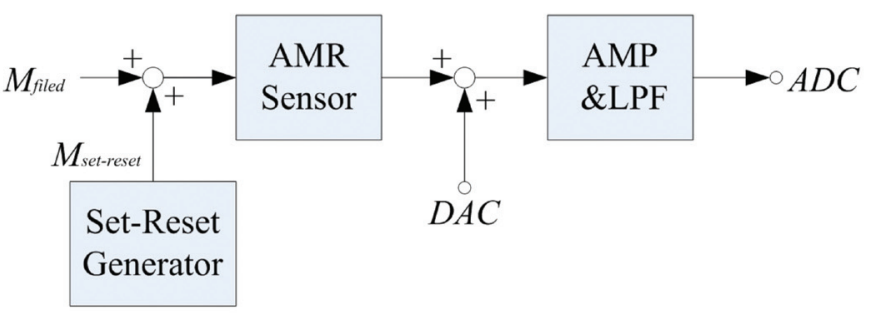

Fig. 7. (a) Four AMR resistors are connected as Wheatstone bridge to form an AMR sensor (Courtesy of Honeywell International, Inc.). (b) Block diagram of a one-dimension AMR circuit.

ture sensor in the vicinity of the AMR sensors to measure the temperature of the AMR.

\subsection{Electron Energy Spectrometer}

A polar orbiting satellite, such as Tatiana-2, at ionospheric altitude provides us with a great opportunity to study the auroral particle dynamics that dominate space weather features at high latitudes. For the ESEMS project, our primary goal is to build the simplest particle instrument (e.g., an electrostatic analyzer) as the starting point for developing our capability in space instrumentation for more sophisticated particle detectors for future missions. The design of the proposed auroral electron energy spectrometer (EES) for the NCU-payload is similar to that of the SSJ/4 electron detectors onboard DMSP satellites (e.g., Hardy et al. 1984), but with reduced energy channels in the energy range between $20 \mathrm{eV}$ and $20 \mathrm{keV}$. As shown in Fig. 8, the principal components of the EES consist of a collimator, a pair of electron deflection plates and a channel electron multiplier (CEM). The collimator is made of aluminium alloy with an electron inlet and an electron outlet along the axis perpendicular to the inlet. The voltage potential of the collimator is positive with respect to ambient plasma. As the positive voltage rejects positive ions and absorbs the electrons that do not move along the axis, the collimator allows only the axis aligned electrons to pass through. The deflection plate set contains two concentric curved aluminium plates. The stepped potential differences are applied to the two aluminium alloy plates to create stepped electric fields in the space between the two plates. If the incident electron's energy is such that the centripetal force experienced by the electron as its trajectory is bent by the electric field equals the electric force, the electron passes along the gap between the plates and strikes the front end of the CEM. As the tangential direction of the arc at the exit of the deflection plates is overlapping with the axis direction of the CEM, the energy-selected electrons are fed into the CEM and then are amplified to generate the pulse signals to be counted. All the other electrons are either absorbed by the collimator or corrupted into the deflection plates.

The EES applies various steps of electric field to the electron deflection plates. After the settlement of each stepped electric field, the counter starts to count the electron incident events from the output of the channel electron multiplier (CEM) for a period about $100 \mathrm{~ms}$. The proposed auroral electron energy spectrometer (EES) for the NCUpayload was designed to measure precipitating electrons

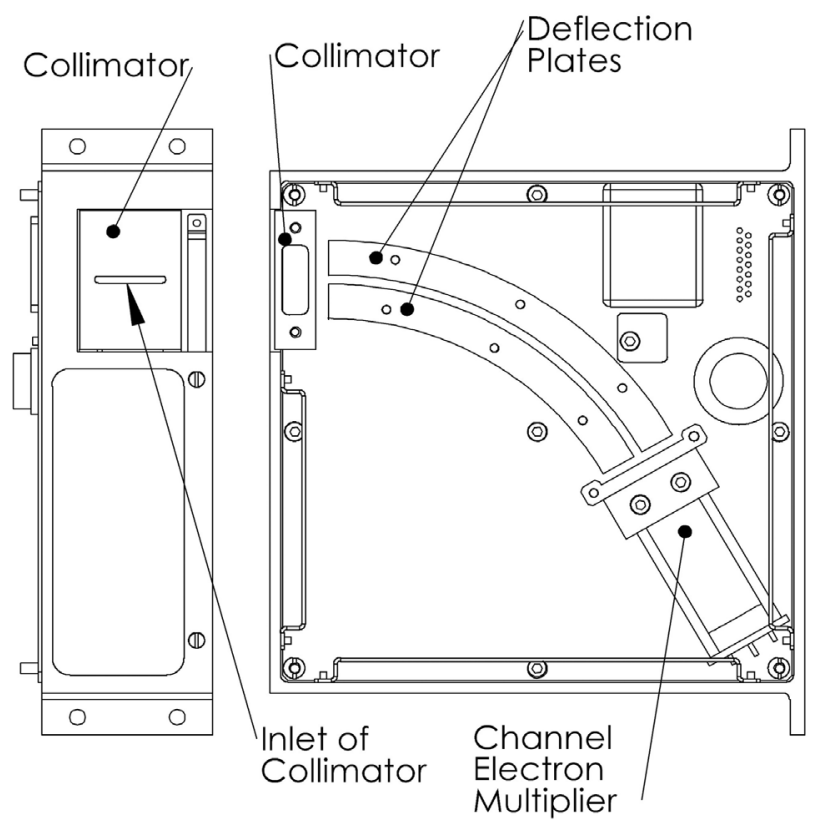

Fig. 8. Schematics diagram of an electron energy spectrometer (EES). 
in 10 channels equally spaced logarithmically in an energy range between $20 \mathrm{eV}$ and $20 \mathrm{keV}$. Although the prototype EES was completed as our original plan, we did not further pursue the development of the engineering and the flight modules for EES due to the weight limit of BCU and the schedule constraints.

\section{DESCRIPTION OF SUPPORT SYSTEMS}

To insure the success of scientific tasks and confirm that the operational environment for the sensors is normal, we also provide the data processing unit, power supply, and temperature sensors as subsystems of the BCU payload. We briefly describe each of the subsystems below.

\subsection{Data Processing Unit (DPU)}

The DPU is the brain of the payload cluster and responsible for the sensors' operation, data acquisition, data packaging, data buffering and uploading of data to the satellite.

\subsubsection{Hardware}

Figure 9 is a photo that shows the data processing unit circuit. The data processing unit uses a 16-bit digital signal processor from Analog Devices Inc. with a $160 \mathrm{MHz}$ core clock frequency and $128 \mathrm{~KB}$ internal memory. The range of operating temperature is from -40 to $85^{\circ} \mathrm{C}$. The DPU consists of a built-in 8-channel/14-bit analog-to-digital converter (ADC), serial peripheral interface (SPI) ports, digital I/O and RS-422 communication interface ports. There are external components on the circuit board including $4 \mathrm{MB}$ static memory (SRAM), 6.4 MB flash memory, a pair of one to six analog multiplexers (MUX) for temperature sensors, and an 8-channel/12-bit digital-to-analog converter (DAC). It takes up 2 and 3 ADC channels, respectively, for the outputs of the ETP and MRM measurements. Through the multiplexer, the six temperature sensors share one ADC channel. The MRM and EES each take three and one DAC channel, respectively. The MRM uses them to adjust the offsets and the EES uses it to change the channel voltage. The DPU also uses digital outputs to control the measurement phases of the equipments. To perform the ETP sinusoidal amplitude switching, two digital outputs are used. The MRM Set-Rest current impulse control takes one digital I/O. The EES event counter requires one digital input.

\subsubsection{Command \& Data Handling}

The data measured by the scientific payloads should be transmitted to the ground via satellite telemetry for post-flight analysis. In order to improve the autonomy of

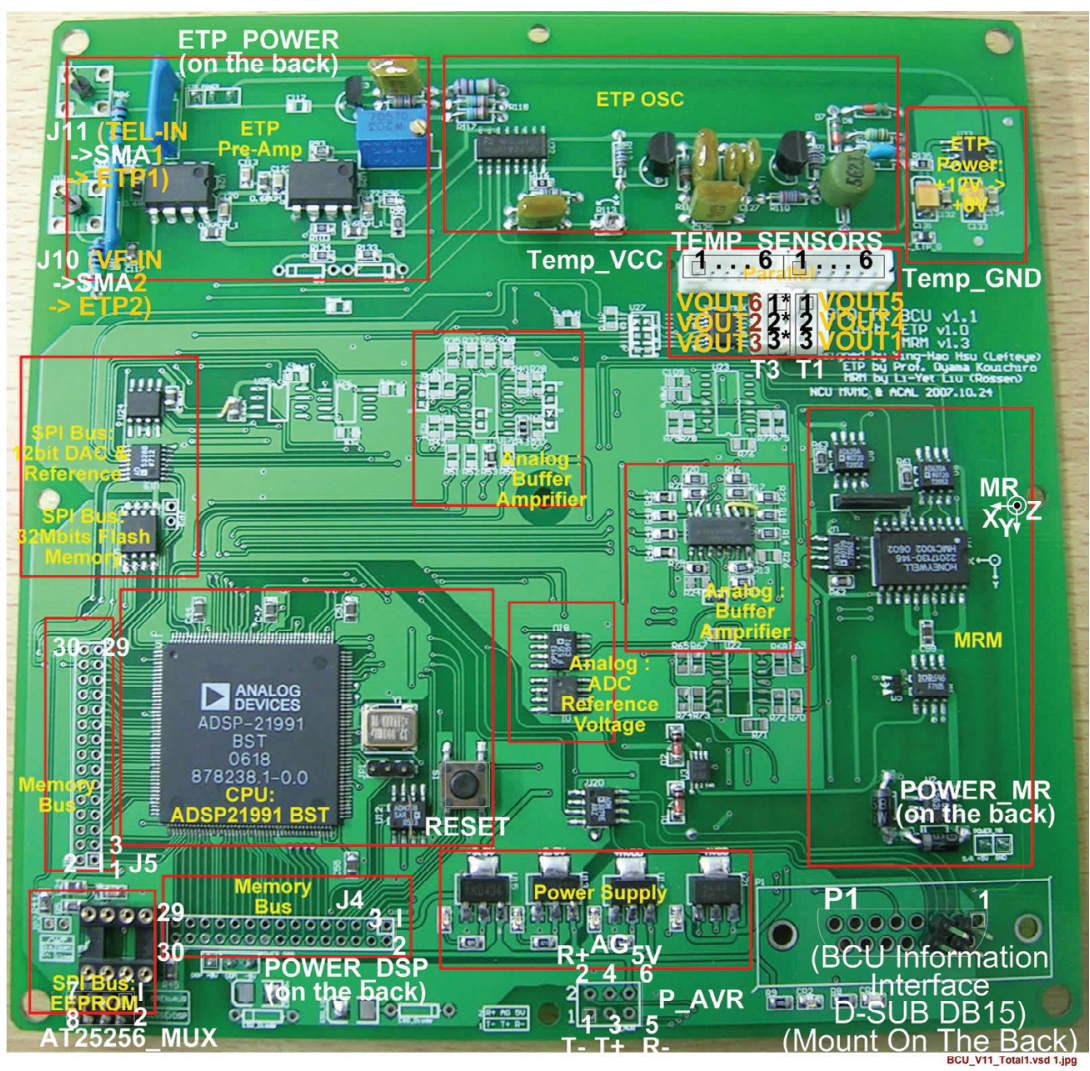

Fig. 9. Circuit board of the data processing unit. 
scientific measurement by the BCU and to minimize the dependency of the BCU on the satellite's commands for instrument operation and data handling, our payloads have its own data processing unit and sufficient buffer memory to buffer the data to be collected. Furthermore, we provide serial communication commands for the Tatiana-2 BI (block of information) module to read the data from the BCU buffer memory. Altogether BCU provides 10 commands to interface with the BI. They are: Sampling On, Sampling Off, Calendar Time Synchronizing, Calendar Request, Flash Buffer Memory Clear, Ready for Power Off, Data Upload, Sensor Reset, Sensor Report, and Error Reports.

Figure 10 shows the formats of the communication packet and data stream. The first line is the communication packet format that has five fields which include the packet header, command, packet length, data, and checksum. Both the command packet and the response packet follow the same packet format. The data field can be the command parameters or the down link data string. The data string in the buffer memory can be directly put into the data field to be down link. The BCU packages the information collected and stores them in the flash memory buffer, waiting for the next down link. Both science data and health information of the BCU are recorded. These data are packed into the formats of Fig. 10, in that the Sampling Data Sequences and the System Data Sequences are shown, respectively, on the second and the third lines. Both of the two data sequences contain a time tag to facilitate data processing to exclude corrupted data and to confirm that only the correct information is used. The time tags enable us to reconstruct the timing of the right packets after the elimination of the corrupt packets.

The Sampling Data Sequence contains scientific data measured every $0.45 \mathrm{sec}$ cycle: the ETP measurement data, MRM measurement data and associated temperature sensor data. Each System Data Sequence contains system health parameters, and the data of the temperature sensors, which are measured every minute.

The BCU flash memory has a 6.4 MB capacity, which is able to buffer a record length of 1039 minutes, or equivalently spanning 0.72 days (or 10.2 orbits) worth of $\mathrm{BCU}$ data. When the satellite is scheduled to downlink the BCU data, the Tatiana-2 BI can issue the data upload command to request those data from the $\mathrm{BCU}$. The $\mathrm{BCU}$ will send back three packets of data every 7 seconds, by way of the satellite downlink to the ground station.

\subsubsection{Sequential Control of Measurement}

When the BCU performs the functions of power converters, Set-Reset function of the MRM, switching amplitude of ETP, and voltage adjustment of EES electric field, large pulses can be produced, which can easily interfere with the measurement signals. The signals to be measured by the BCU sensors are usually weak and need to be enlarged before sampling. At the same time of amplifying the measurement signals, however, the interference noises may also be amplified. Since all electronics of the BCU are packaged in the same chassis, the possibility of the interferences resulting from those operations is high. To eliminate the interference and improve the accuracy, we have implemented a two-layer structure in the chassis to isolate the instruments. In addition, we have carefully arranged the timeline for performing various tasks in which the measurement timing is separated from the switch timing in order to exclude the unnecessary interactions.

As shown in Fig. 11, we define a basic cycle period, T_Base $=150 \mathrm{~ms}$, and arrange all the switch actions such as Set-Reset which will generate pulse signals to be performed at the time of the successive T_Base cycle changes. Thus for the ETP to complete a measurement cycle, it will require three basic cycles to set the amplitude of the sinusoidal at $0.5,0.25$, and $0 \mathrm{~V}$, respectively. While for EES to complete a measurement cycle, we need ten basic cycles to set up the 10 different voltages (electric fields) between the electron deflected plates.

Because the time needed to complete one MRM measurement including the Set-Reset and data acquisition is much shorter (just about $125 \mu \mathrm{s}$ ) than those of the other two instruments, we arrange one MRM measurement at the beginning of each ETP measurement cycle to obtain a
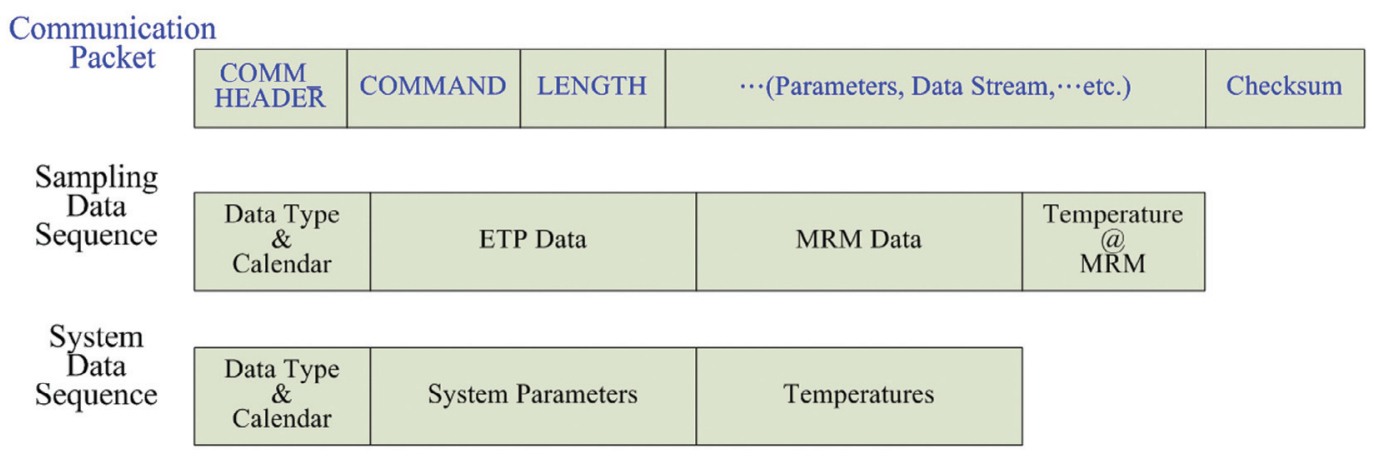

Fig. 10. Communication packet and Data Sequence Format. 


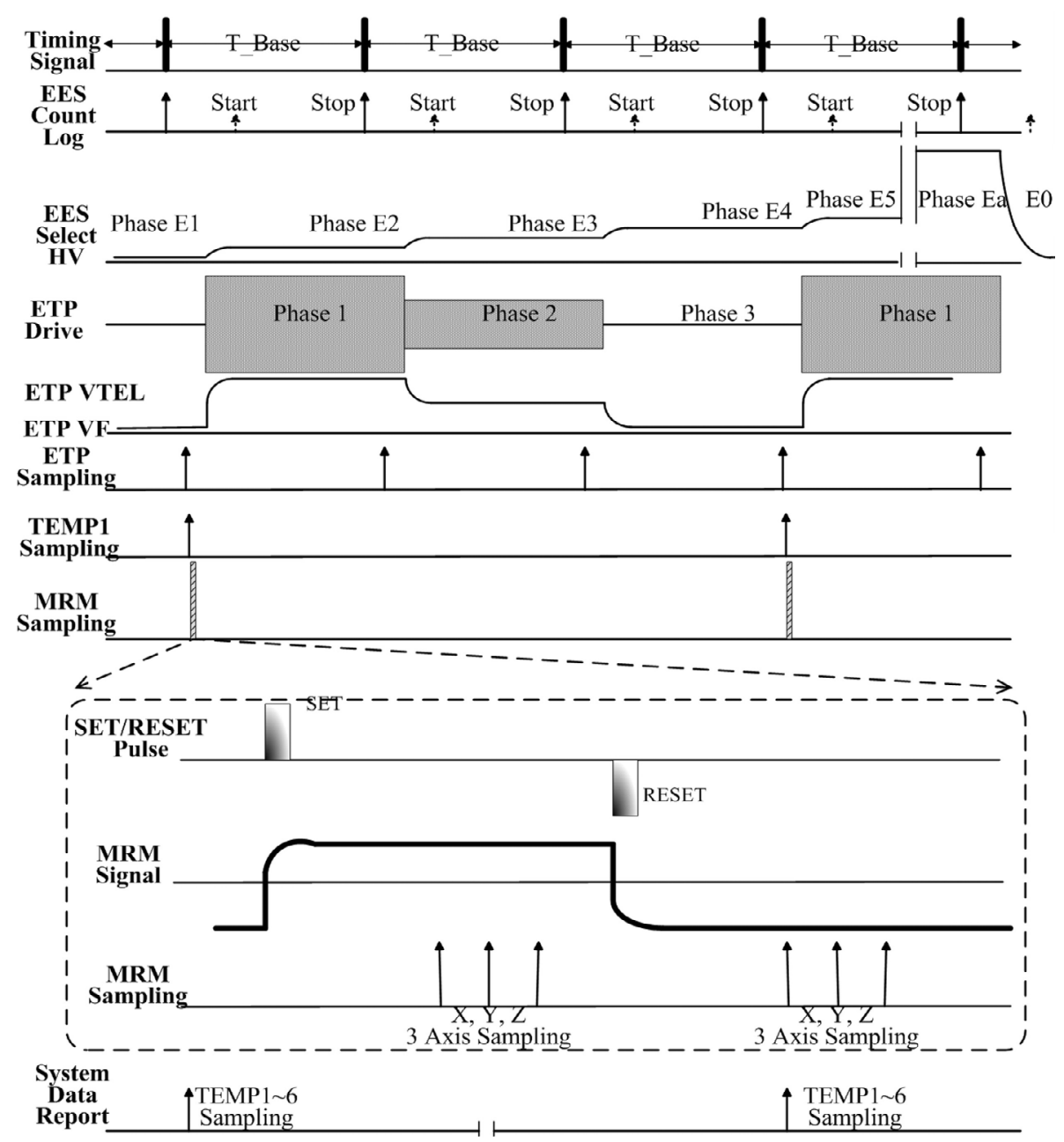

Fig. 11. Timing sequence of data sampling in BCU.

down-graded and synchronized $450 \mathrm{~ms}$ cycle period. It should be noted that only after the completion of the above actions of the MRM, the phase switching actions of the EES and ETP are performed, and magnetic vector has been acquired in the meantime. The ambient temperature inside the $\mathrm{BCU}$ chassis is also measured, which provides information for temperature compensation/correction as required by the scientists to post analyze the MRM data.

\subsubsection{Flight Software}

As previously mentioned command and data handling $(\mathrm{C} \& D H)$, and sequential control of measurement (SCM) are all performed by the DPU with the inflight software. The code block of the flight software that is responsible for the C\&DH is separated from the SCM. However, both blocks rely on the state and control flags. The C\&DH block is able to control the operation of SCM, so that these two code blocks work together to complete the scientific mission of the BCU.

C\&DH actions are launched by communication, and one communication involves dozens to hundreds serial port reception events. There are two programming styles that we commonly use to handle the reception events. The first uses the serial port hardware interrupt service routine, and the second polls the serial input flag under the main program. The left panel of Fig. 12 shows the main program flow chart, in which the C\&DH program of our system uses the polling method. By polling the serial input flag when there is new data, the main program reads the Rx for data and cumulates the data to form a packet. When the accumulation of the full packet is completed, the packet is sent to the command process to decode the instruction(s). According to the instruction(s), the process will set or clear the control flags to modify the operation modes of the BCU system. Regardless of the packet completeness, if error occurs in the accumulation, the program will send back a "communication error" response. If the instruction says "power off," the program will store all the experimental data that are still in RAM buffer into the flash memory with their associated sampling times, and switch the system state to power off.

The right panel of Fig. 12 shows the timer interrupt service routine (ISR). In the routine, the execution of the 


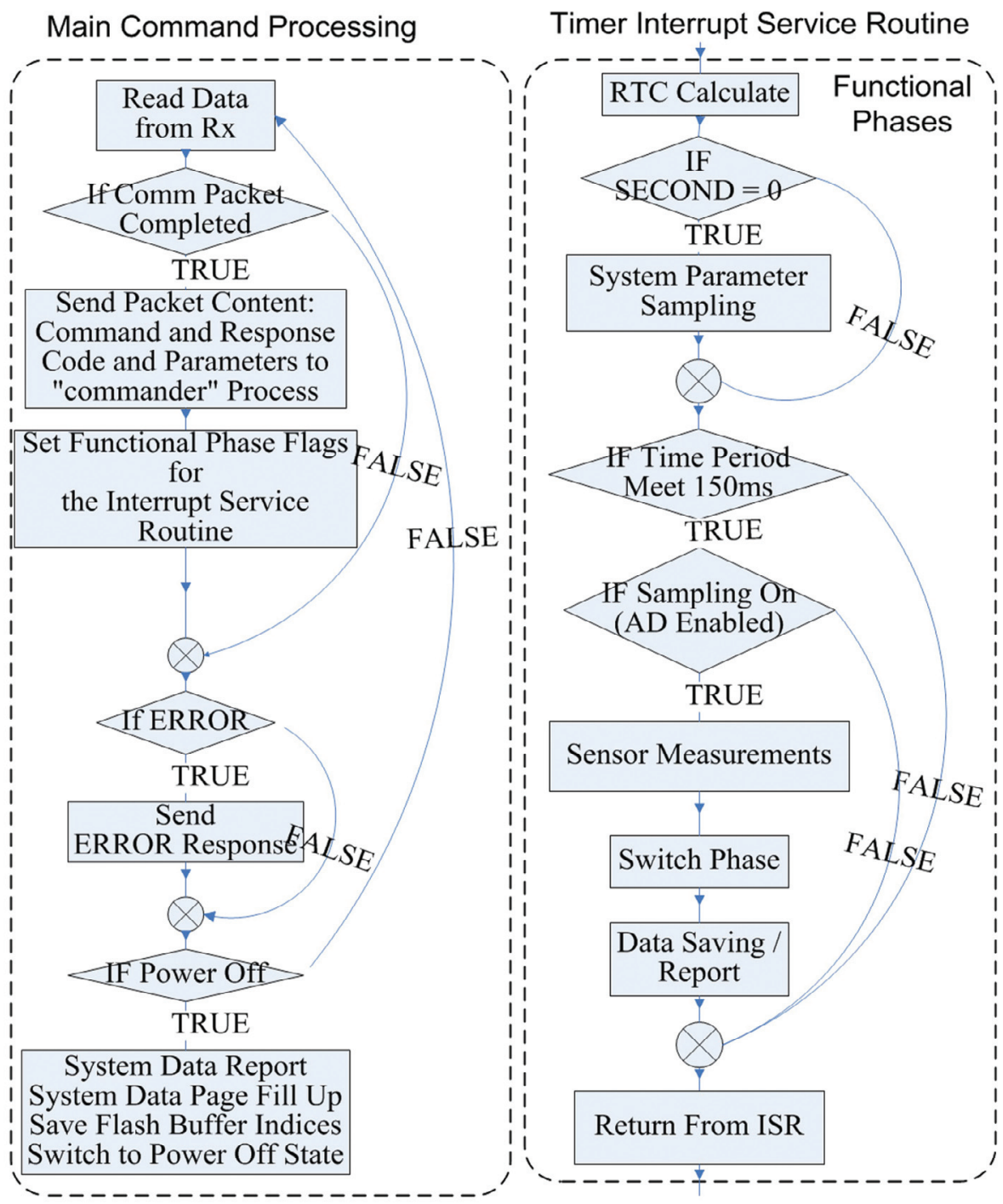

Fig. 12. Flow chart of flight software.

SCM program is triggered by a timer interrupt. System parameter sampling and sensor data measurements share the same hardware timer interrupt. When the timer interrupt occurs, the ISR checks the real time clock (RTC) to see if the sec-reading is zero to determine the one-minute period for recording the system parameters every minute. In addition, ISR can detect the $150 \mathrm{~ms}$ period by successively combining software counter and hardware timer. Timing the $150 \mathrm{~ms}$ period is important since during every $150 \mathrm{~ms}$ cycle the DPU will execute the instruments phase switching and the sensors data sampling.

\subsection{Miscellaneous}

In addition to the major scientific instruments and DPU computer, the $\mathrm{BCU}$ system also includes the $\mathrm{DC} / \mathrm{DC}$ power supply to support the scientific instruments and computer power, temperature sensors to measure the temperature of the instruments and computers to verify that the system is operating under normal working conditions.

The BCU has three sets of power converters to convert the satellite provided $28 \sim 34 \mathrm{~V}$ power to $+12,+5$, and -5 $\mathrm{V}$, respectively, as the required energy sources for various BCU operations. Specifically, the $+12 \mathrm{~V}$ power is for ETP and EES. While the +5 and $-5 \mathrm{~V}$ are applied to the MRM, DPU, and peripheral circuits. The allocated power that the satellite provides is $10 \mathrm{Watts}$, and the surge current tolerance is 10 amps. While the required power consumptions for the BCU modules are $0.4,0.5,2$, and $1.6 \mathrm{~W}$, respectively, for ETP, MRM, DPU and other on-board peripherals. The three sets of power converters can provide power (and its maximum current) of $12 \mathrm{~V}(100 \mathrm{~mA}), 5 \mathrm{~V}(1 \mathrm{~A})$, and $-5 \mathrm{~V}(100 \mathrm{~mA})$ to meet the requirements of each of the $\mathrm{BCU}$ modules. The requirement that the total power consumption of the BCU is less than $80 \%$ of the power supplied by Tatiana-2, allows our power conversion circuit to work 
under the worst conversion efficiency which is larger than $80 \%$. The measured total power consumption of the BCU flight module is $4.5 \mathrm{~W}$, and its power surge current is 8.64 A, which are all within the specified ranges of the satellite's power supply.

BCU uses six identical RTD temperature sensors to measure the temperature at the six sides of the bulkhead of the BCU chassis. The sensors can measure temperature ranging from -40 to $125^{\circ} \mathrm{C}$. The sensors are small, each with a size of about $14 \mathrm{~mm}$ long $\times 12 \mathrm{~mm}$ wide $\times 2.5 \mathrm{~mm}$ thick. They can be directly fixed to the aluminium bulkhead surface with screws.

\subsection{Development Tools}

In addition to the implementation of circuit and mechanical structure within the body of the scientific payload, we have designed two tools, including a plug-in programming loader and a serial communication listener for development and testing. With these tools we are able to improve the program's development as well as later modifications, and the efficiency of satellite-payload interface testing without worrying much about the language challenges, travel time limits, and financial constraints between our international collaborators.

The main purpose of designing a plug-in program loader is that we need to regularly update the DPU firmware during the process of developing the BCU payload. The loader consists of two ports. One is connected to the BCU's burning port. The other port is connected to the PC through the RS232-to-USB converter. Therefore users can develop programs on a personal computer and load the program into BCU to achieve the update purpose. When the tasks of development and interface testing are completed the program loader can be removed, which neither increases the weight of the payload nor affects the power consumption.

The communication interface between $\mathrm{BCU}$ and Tatiana-2 is done via a RS-422 port. If one can monitor the bi-directional communication strings between them, the problems in commands or responses can be quickly identified and fixed during the integration test. For this purpose, we have developed a serial communication listener to listen to the RS-422 communication between Tatiana- $2 \mathrm{BI}$ and the $\mathrm{BCU}$, then record and display contents of both sides on a PC screen. In fact we have applied this monitoring tool to the BCU and Tatiana-2 integration test. As a result, the satellite developers and our testing staff have effectively resolved the associated problems encountered in the communication within a half day.

\section{IN-FLIGHT DATA}

With the successful launch of Tatiana-2 into the target orbit, the onboard BCU payload has survived the harsh en- vironment of launch and successfully operated in the near Earth space environment. The clearest evidence of these facts is the real space in-flight data. The flight data of BCU will be presented with verifications by their nominal features in the following. However, the purpose of this paper is to verify the successful implementation of the engineering tasks of the BCU, the scientific interpretation and their scientific significance will be published by the BCU scientists elsewhere.

Figure 13 shows the comparison of the magnetic field measurements by our MRM at a sampling rate of $2.2 \mathrm{~Hz}$ with those by the magnetometer of the Technical Master (TM) of the Tatiana- 2 at a sampling rate of $1 / 60 \mathrm{~Hz}$ (1 minute) for the period of 21:36 - 08:24 UT on 19 - 20 December 2009. Since both magnetometers are attached to the same satellite in the SBC coordinates, their data can be checked against each other for verification. As shown, the two sets of data agree closely in both $B_{x}$ (horizontal-right) and $B_{y}$ (nadir) components. The variations of the two sets in the $\mathrm{B}_{z}$ (ram) component resemble each other coherently. However, there exists an offset about 0.146 Gauss between the two. As the Tatiana- 2 magnetometer could have been calibrated to remove the inherent offset in the satellite body, the offset reflects that the operational environment of our payload has a strong bias magnetic field in the ram direction. The TM magnetometer data can be used as our reference for in-flight calibration.

Figures 14 and 15 show, respectively, the in-flight data of the MRM and ETP on board the Tatian- 2 on 18 January 2010 during 00:28:48 03:50:24 UT. These data are plotted according to UT time with references to geographic latitude GLAT, longitude GLON, and local time LT at the location of the satellite. The satellite travels southbound in daylight at nominal $8.93 \mathrm{hr}$ LT and evening after dark at $20.93 \mathrm{hr}$ LT during northbound.

In Fig. 14, the in-flight calibrated MRM data are plotted in comparison with the nominal values derived from the International Geomagnetic Reference Field model (IGRF). The magnetic field vectors are described in terms of the three orthogonal components in the geographic coordinate system (namely east-west $\mathbf{B}_{\mathrm{E}-\mathrm{w}}$, north-south $\mathbf{B}_{\mathrm{N}-\mathrm{S}}$ and up-down $\mathbf{B}_{\mathrm{U}-\mathrm{D}}$ components). In general the two data sets are quite consistent. As shown, at equatorial latitudes both $\mathbf{B}_{\mathrm{N}-\mathrm{S}}$ components show the peak values and are all positive (northward), while the $\mathbf{B}_{\mathrm{U}-\mathrm{D}}$ components have the minimum magnitudes. While at polar latitudes the $\mathbf{B}_{\mathrm{U}-\mathrm{D}}$ components have the maximum values. The $\mathbf{B}_{\mathrm{U}-\mathrm{D}}$ is downward in the northern polar region and is upward in the southern polar region. There are deviations between IGRF and MRM data for the two $\mathbf{B}_{\mathrm{N}-\mathrm{S}}$ and $\mathbf{B}_{\mathrm{E}-\mathrm{w}}$ components, which may reflect the existence of ionospheric currents. Based on the MRM data taken at high latitudes, Liu et al. (2012) have derived the field-aligned currents of typical magnitude from the perturbations in the $\mathbf{B}_{\mathrm{N}-\mathrm{S}}$ and $\mathbf{B}_{\mathrm{E}-\mathrm{w}}$ components. 
The floating potential measurements of ETP are presented in Fig. 15. As mentioned in section 3.1 the floating potential of a probe is determined by the current balance condition at the probe. The operation of the floating ETP relies on the fact the mean potential of a floating probe with respect to the ambient plasma will be shifted negatively if a sine wave voltage is applied to it. Equation (1) shows that the amount of floating potential shift $\left(\Delta \mathrm{V}_{\mathrm{f}}\right)$ is related to the
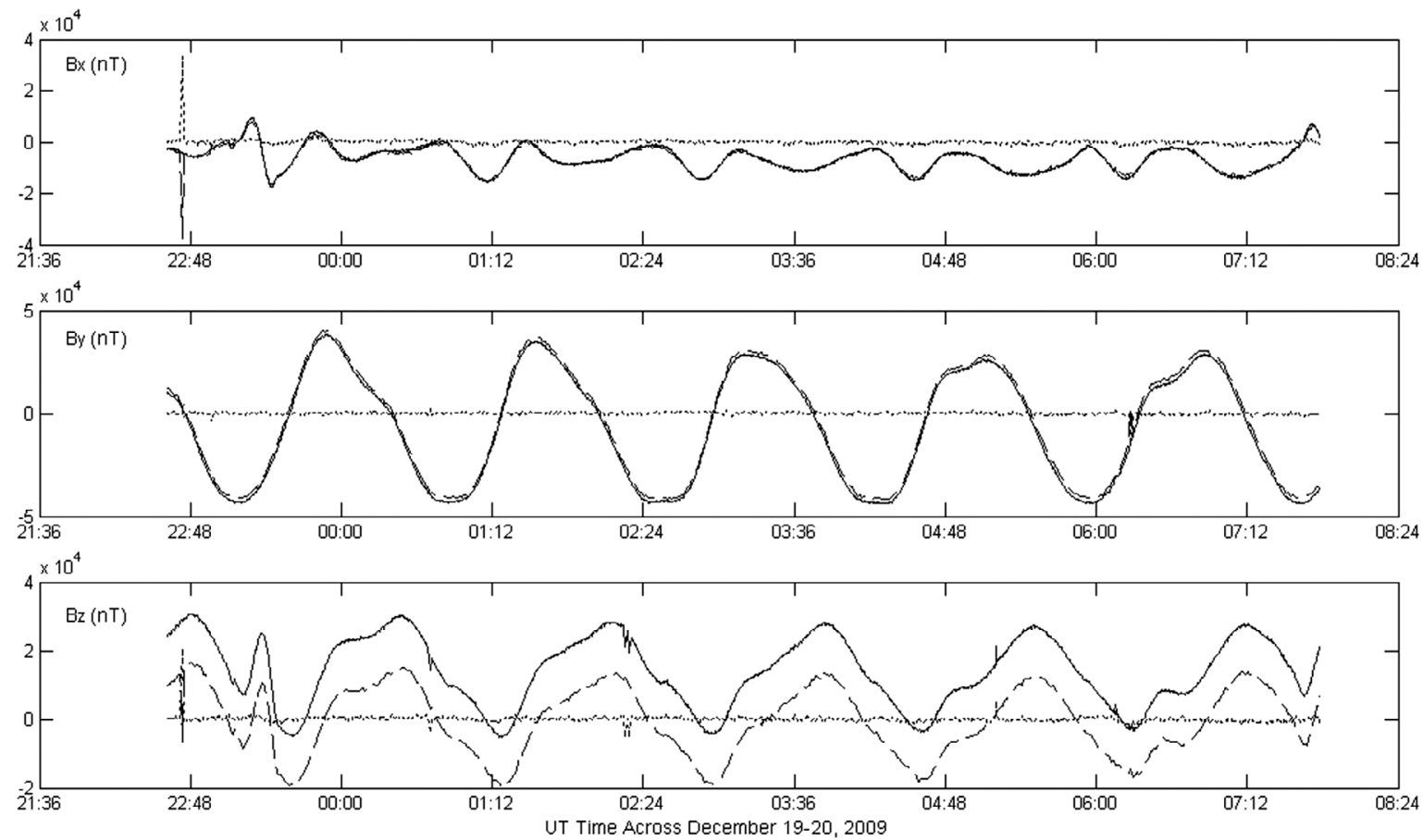

Fig. 13. Comparison of in-flight data of the MRM (solid line) with that of the Tatiana-2 TM magnetometer (dashed line) in the SBC coordinate system: $\mathrm{z}$ - ram, $\mathrm{y}$ - nadir, $\mathrm{x}$ - horizontal right.

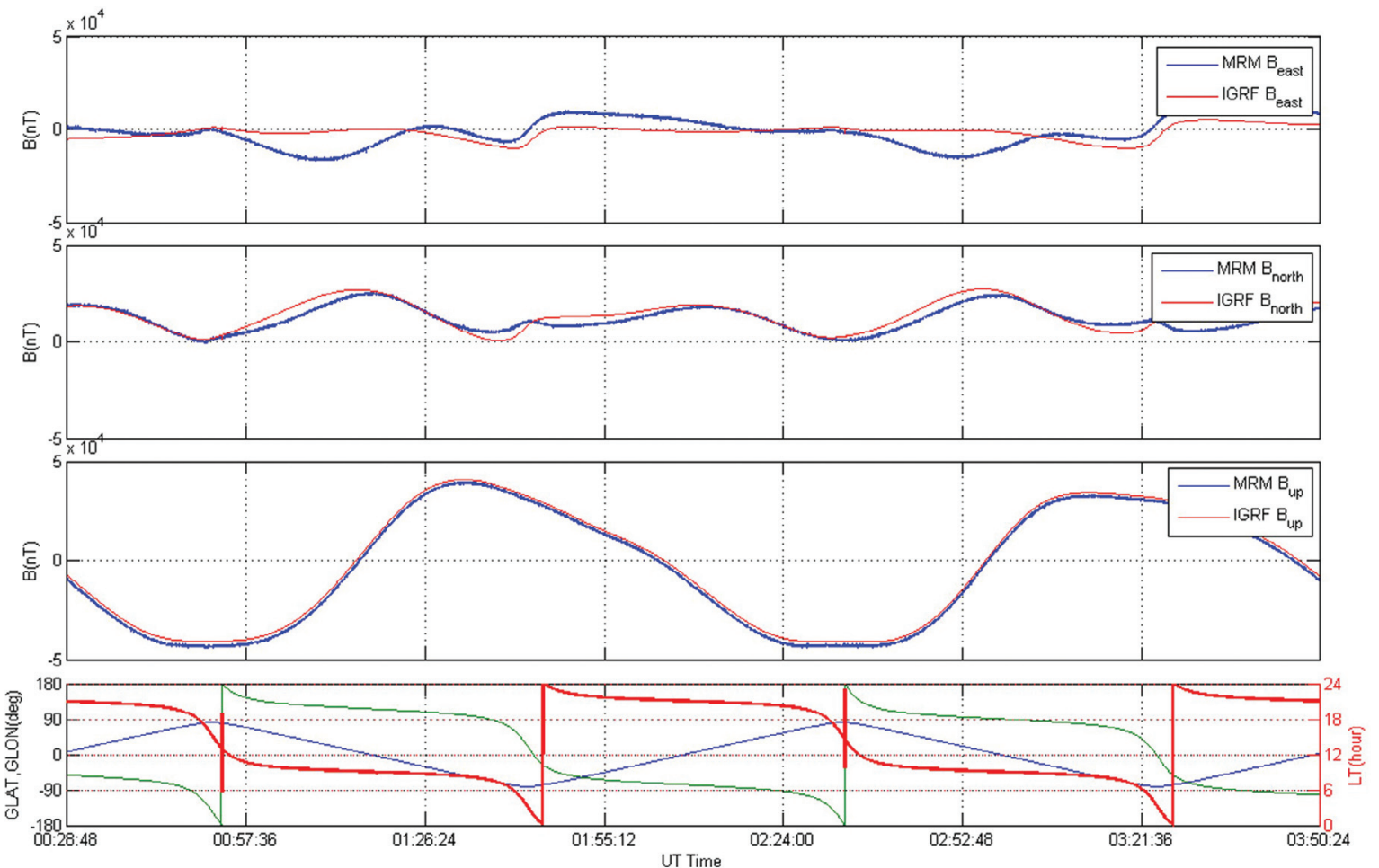

Fig. 14. Comparison of an in-flight calibrated MRM data on date 18 January 2010 with the nominal IGRF. 


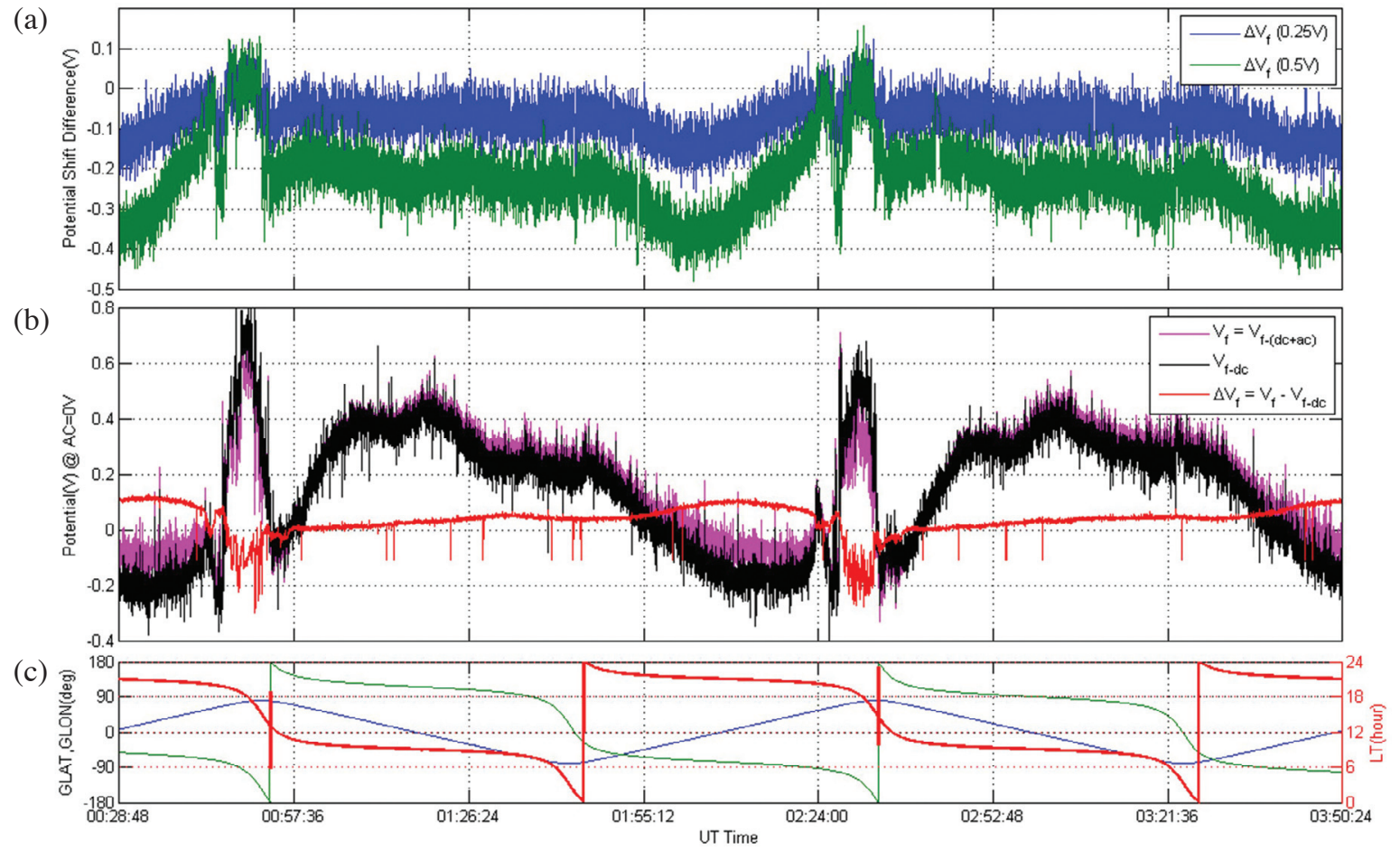

Fig. 15. ETP in-flight measurement on date 18 January 2010. (a) Floating potential shifts measured during the two phases of a $=05 \mathrm{~V}$ and $\mathrm{a}=0.25$ V; (b) Floating potentials measured by the two electrodes, their differences are in red; (c) the satellite location in geographic latitude and logitude, and local time coordinates.

amplitude [a] of the sine wave voltage and the temperature $\left(\mathrm{T}_{\mathrm{e}}\right)$ of the Maxwellian-distributed ambient electrons. As indicated in the equation, " $\Delta \mathrm{V}_{\mathrm{f}}$ " increases with increasing " $\mathrm{a}$ " when " $T_{e}$ " is constant, while " $\Delta V_{f}$ " decreases with increasing " $T_{e}$ " for the fixed "a" condition. For a known "a", the electron temperature can be derived from Eq. (1).

In our case we have applied "a" to the measurementelectrode plate (P) of ETP in three phases: (1) a $=0.5 \mathrm{~V}$; (2) $\mathrm{a}=0.25 \mathrm{~V}$; and (3) $\mathrm{a}=0 \mathrm{~V}$. By applying $\mathrm{a}=0 \mathrm{~V}$ to $\mathrm{P}$, the biases between the outputs of the reference-electrode $\left(\mathrm{P}_{\text {ref }}\right)$ and those of the measurement-electrode can be resolved, which will allow us to perform an in-flight calibration of the ETP. The floating potentials measured by the two electrode plates during the phase of $\mathrm{a}=0 \mathrm{~V}$ are presented in the second panel of Fig. 15, while the calibrated floating potential shifts measured during the other two phases are presented in the top panel. As seen from the second panel the two electrode plates measured negative floating potential when the satellite travelled northbound through the night side ionosphere until the satellite arrived at the northern polar region where positive floating potentials are detected and differences between the two sets of floating potential are pronounced (see the red curve). In juxtaposition to this observation, positive floating potentials were observed when the satellite travelled southbound to even over the southern polar region in the sunlit ionosphere (recall that the data were taken on a southern summer day of 18 January 2010).
In night side auroral latitudes, precipitating high energy particles and their associated secondary electrons can play a dominant role in the current balance to the ETP and in the energy budget of the ionospheric plasma. As a result, positive floating potential and high temperature plasma may be detected by ETP. On the other hand, solar radiation can warm up the sunlit ionosphere and generate the photoelectrons backscattered from the ETP. The backscattered photoelectrons can play a significant role in the current balance condition to produce positive floating potentials in the day time ionosphere. Examining the ETP data in the top panel, we found that the ETP measured the colder $T_{e}$ through the night side of the ionosphere, which is revealed by the larger difference between the two sets of floating potential shift. Warmer $T_{e}$ regions were found in the sunlit ionosphere as indicated by the smaller differences between the two sets of $\Delta \mathrm{V}_{\mathrm{f}}$ measurements. However, the hottest $\mathrm{T}_{\mathrm{e}}$ was found in the auroral region as revealed by the two undistinguished small $\Delta V_{f}$ measurements. These features further support the above interpretations of the ETP observations. In summary, the in-flight data of Fig. 15 have confirmed that the BCUETP instrument has been functioning well in space.

\section{CONCLUSIONS}

In this paper we report the engineering efforts in completing a space qualified BCU payload on board the Tati- 
ana-2 satellite. The BCU payload is not only the first scientific satellite payload designed and built entirely by the team from National Central University in Taiwan, but also the first Taiwanese scientific payload to be placed aboard a Russian satellite. Preliminary results of the down linked BCU data have verified that the design and operation of the BCU payload met the requirements for space missions.

The major steps to develop a scientific satellite payload included (1) sensor selection to meet scientific objectives; (2) design of the main electronic package to control and operate the sensors and to interface with the satellite subsystems; (3) fabrication of the flight module of a payload according to the designs; (4) pre-flight payload calibration (see Liu et al. 2012 for the detailed MRM calibration); (5) payload-satellite integration tests; and (6) scientific application of the payload. By means of the BCU project, we have gone through all the required engineering steps for building a space-qualified payload.

We originally proposed EES, ETP and MRM as the instrument package to measure the particle and field features throughout the topside ionosphere for space weather investigation. Because of weight constraints of the BCU and need to meet the satellite launch schedule, we withdrew the EES from the further development of flight module. We therefore concentrated on the implementations of a miniature magnetometer and an electron temperature probe which has the similar design to those of Japanese ETP for the flight module of BCU.

The instruments of the ETP and MRM were integrated with a DPU into the main electronic package (MEP) which is controlled by the in-house developed flight software. The BCU MEP performed very well in flight operations. The tools developed to process the downlink data and to rebuild its timing are convenient and flexible in verifying the mechanisms of timestamp, data buffering, and data conversion for post-flight data analysis.

Finally, the BCU is an international collaborative project in developing the scientific satellite payload between a non-English speaking country and Taiwan. The satellitepayload integration was accomplished successfully within a very limited time constraint. The result verifies that the scheme of satellite-payload interface used by the BCU team can indeed speed up the integration of the satellite with its payloads. This experience establishes a good reference for other international cooperation projects. The lessons learned and experience gained from this BCU project have greatly enhanced our capabilities in developing a scientific payload for future satellite missions.

Acknowledgements The BCU Team appreciates engineering assistance on packaging for survivability through the "Interuniversity Scientific Education Program of Space Research." We are grateful for the grant 95-NSPO(B)-SE-FA07 -02 and are indebted to continual support from the National
Science Council - Science Education Division (NSC-SE) and National Space Organization (NSPO). This support nurtured one and a half decades of effort in the development of students' capabilities in the Opto-Mechanics Engineering (OME) design and implementation program at NCU. Without such an educational system, this project would not have been possible. We are also greatly in debt to Prof Koh-Ichiro Oyama for his passionate encouragement and his guidance in the ETP technology.

\section{REFERENCES}

Caruso, M. J., T. Bratland, C. H. Smith and R. Schneider, 1999: Anisotropic magnetoresistive sensors: Theory and applications. Sensors, 16.

Hardy, D. A., L. K. Schmitt, M. S. Gussenhoven, F. J. Marshall, H. C. Yeh, T. L. Schumaker, A. Huber, and J. Pantazis, 1984: Precipitating electron and ion detectors (SSJ/4) for the block 5D/flights 6-10 DMSP satellites: Calibration and data presentation. AFGL-TR, 84-0317, ADA157080, Air Force Geophysics Laboratory, Hanscom AFB, Mass., No. 902.

Hirao, K. and K. Oyama, 1970a: Effect of electron heating on the electron temperature probe. J. Geomag. Geoelectr., 22, 341-352.

Hirao, K. and K. Oyama, 1970b: An improved type of electron temperature probe. J. Geomag. Geoelectr., 22, 393-402.

Honeywell International, Inc., 2000: 1- and 2-Axis Magnetic Sensors (Datasheet), available at http://www.magne ticsensors.com/literature.php.

Hsu, Y. H., L. Y. Liu, C. J. Chou, J. W. Jiang, J. Y. Peng, S. B. Jiang, H. C. Yeh and T. L. Yeh, 2008: ESEMS - AMEI BCU: The engineering architecture, $4^{\text {th }}$ Asian Space Conference, Taipei, Taiwan.

Kalegaev, V., 2009: Tatiana-2, Space Monitoring Data Center Skobeltsyn Institute of Nuclear Physics, Moscow State University, Russia, available at http://smdc.sinp. msu.ru/index.py?nav=tat2.

Liu, L. Y., S. B. Jiang, T. L. Yeh, H. C. Yeh, J. Y. Liu, Y. H. Hsu, and J. Y. Peng, 2012: The magneto-resistive magnetometer of BCU on the Tatiana-2 satellite. Terr. Atmos. Ocean. Sci., 23, doi: 10.3319/TAO.2011. 11.07.01(AA), in press. [Link]

MsuNews, 2010: The orientation system of the university satellite Tatiana- 2 is out of service. MsuNews.ru, 2010, February 16, available at http://www.msunews. ru/news/2499/.

Oyama, K., 1976: A systematic investigation of several phenomena associated with contaminated langmuir probes. Planet. Space Sci., 24, 183-190, doi: 10.1016/00320633(76)90104-5. [Link]

Oyama, K., 2008: Technical information of electron tem- 
perature probe (ETP). NCU Space Science technical note ETP TEC2008-3/11.

Oyama, K., Y. Kakinami, J. Y. Liu, M. Kamogawa, and T. Kodama, 2008: Reduction of electron temperature in low-latitude ionosphere at $600 \mathrm{~km}$ before and after large earthquakes. J. Geophys. Res., 113, A11317, doi: 10.1029/2008JA013367. [Link]

Yeh, H. C., S. B. Jiang, J. Y. Liu, and T. L. Yeh, 2008a:
Development and application of space weather instrument on experimental scientific-education microsatellite (ESEMS), NCU-Final Report for the project 95-NSPO(B)-SE-FA07-02.

Yeh, T. L., S. B. Jiang, H. C. Yeh, J. Y. Liu, K. Oyama, Y. H. Hsu, L. Y. Liu, C. J. Chou, J. W. Jiang, and J. Y. Peng, 2008b: ESEMS - AMEI BCU, $4^{\text {th }}$ Asian Space Conference, Taipei, Taiwan. 